adjoining hollow in the belt and of the equatoreal spots have conformed very closely with the rates upon which systems I and II are based in the ephemerides alluded to.

The dark spot in the $\mathrm{N}$-tropical zone of Jupiter mentioned by J. Comas Solá, Astr. Nachr. 3719, and by the Rev. T. E. R. Phillips, Astr. Nachr. 3724, was of very temporary character for it rapidly grew faint and disappeared altogether.

Many observers will probably have remarked a dark mass or irregular group of spots lying on the southern borders of the great southern equatorial belt. This has recently formed a prominent feature and was situated in longitude $232: 8$ (system II) on Aug. 3 and in $217^{\circ}: 0$ on Sept. I so that its rotation period is about $9^{\mathrm{h}} 55^{\mathrm{m}} 20^{\mathrm{s}}$. There was a somewhat similar outbreak of spots in the same zone in the early part of June 1889 seen by Mr. A. S. Williams and they formed the subject of an interesting contribution from his pen in Monthly Notices Vol. L, p. 520 to 522 . The more rapid velocity of these objects caused them to overtake the ellipse of the red spot in July 1890 when they were immediately diverted to the south and drifted along the southern edge of the ellipse. - It remains to be seen whether the present outbreak will retain a visible form until its swifter motion enables it to overtake the red spot in about 9 months from the present time viz in 1902 June and whether the experiences of 1890 will be repeated. It is curious that these disturbances occurred at intervals of 12 years and suggestive that if they are recurrent the period is coincident with that of a revolution of Jupiter round the sun.

Bishopston, Bristol, 190 I Sept. 6.

W. F. Denning.

\title{
Observations of meteors in July and August 1901.
}

\section{By W. F. Denning.}

Between July 15 and 24 during 14 hours of watching 95 meteors were seen of which the paths of 72 were registered. Between Aug. 10 and 22, when fortunately there occurred a series of very clear nights, during 34 hours of watching 40 I meteors were observed of which 242 were registered. The Perseids furnished a display perhaps a little stronger than the average and decidedly more of its meteors were noticed on Aug. I I than on Aug. 1o while the maxi- mum was probably attained on the morning of Aug. 13 but at that time the sky was much clouded at Bristol and fair comparisons could not be made. The first Perseids were recognized on July $2 \mathrm{I}$ and the last on Aug. 21 ; the radiant point exhibited the usual movement of about $I^{\circ}$ per day to the ENE. There were a great number of minor showers among them being the following:

\begin{tabular}{|c|c|c|c|c|c|}
\hline Aug. & $10-22$ & $310^{\circ}$ & $+61^{\circ}$ & 11 & Slow \\
\hline July & $15-21$ & 311 & +13 & $5)$ & \\
\hline Aug. & $11-13$ & 312 & +13 & 5 & $\geqslant$ \\
\hline July & $15-20$ & $3 \times 7$ & +31 & 5 & 2 \\
\hline Aug. & $10-22$ & 335 & +1 & 15 & Slowish, faint \\
\hline » & $10-20$ & 335 & +73 & 13 & Rapid, white \\
\hline » & $15-21$ & 345 & 0 & 7 & Rapid \\
\hline 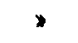 & I $5-19$ & 349 & +48 & 5 & , \\
\hline » & $10-2 I$ & $35^{\circ}$ & -14 & 10 & Slowish \\
\hline . & $10-2 I$ & 353 & $+3 I$ & 6 & , \\
\hline
\end{tabular}

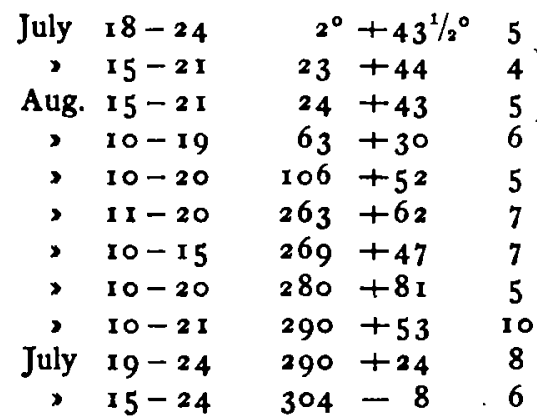

Rapid
Rapid, streaks
Slow
Rapid, short paths
Slow
,

There were other radiants in July at $29^{\circ}+36^{\circ}$ and $20^{\circ}+5^{\circ}$ and in August at $14^{\circ}+7^{\circ}, 33^{\circ}+18^{\circ}, 42^{\circ}+12^{\circ}$, $47^{\circ}+43^{\circ}, 57^{\circ}+9^{\circ}, 71^{\circ}+52^{\circ}$ \&c.

A large number of interesting meteors were doubly observed in England during the period from Aug. IO to 21 and I have computed the following real paths:

\begin{tabular}{|c|c|c|c|c|c|c|c|c|c|c|}
\hline No. & $\begin{array}{l}\text { Date } \\
\text { I } 901\end{array}$ & Gr. M. T. & Mags. & $\begin{array}{c}\text { Height at } \\
\text { beginning } \\
\text { miles }\end{array}$ & $\begin{array}{l}\text { Height at } \\
\text { ending } \\
\text { miles }\end{array}$ & $\begin{array}{c}\text { Length } \\
\text { of Path } \\
\text { miles }\end{array}$ & $\begin{array}{l}\text { Velocity } \\
\text { in miles } \\
\text { per sec. }\end{array}$ & & diant & Observers \\
\hline $\mathbf{I}$ & Aug. 10 & $10^{\mathrm{h}} 4 \mathrm{I}^{\mathrm{m}}$ & $3-2$ & 88 & 68 & 21 & 25 & $278^{\circ}$ & $+67^{\circ}$ & A. S. H., W. F. D. \\
\hline 2 & , & $105^{8}$ & $3-2-2$ & 57 & $5^{2}$ & 25 & 15 & $35^{2}$ & -14 & A.S. H., J.H. B, W. F. D. \\
\hline 3 & $>$ & $12 \quad 01 / 2$ & $\mathbf{I}-\mathbf{I}$ & 76 & $5 \mathrm{I}$ & $3^{6}$ & 50 & 44 & $+5^{81 / 2}$ & A. S. H., W. F.D. \\
\hline 4 & ? & 1216 & $2-\mathbf{I}$ & 69 & 50 & 27 & 39 & 42 & +57 & A. S. H., W. F. D. \\
\hline 5 & > & 1219 & $3-2$ & 72 & 60 & 33 & 25 & 149 & +60 & A.S.H., W.F. D. \\
\hline 6 & , & 1318 & $\mathbf{x}-4$ & 70 & 47 & 31 & 31 & 45 & +56 & O. A: Le Bean, W.F.D. \\
\hline 7 & , & 1334 & $I->I$ & 63 & 29 & 48 & 16 & 269 & +47 & O.A. Le Bean, W. F. D. \\
\hline 8 & Aug. II & I I 2 & $2 \times 9-=D$ & 95 & $5^{6}$ & 64 & v. rapid & 45 & $\begin{array}{l}78 \\
+5^{8}\end{array}$ & D. E. Packer, W.F.D. \\
\hline 9 & , & I i $33^{1 / 8}$ & $4-4$ & 94 & 59 & 55 & 42 & 45 & +57 & D. E.Packer, W.F.D. \\
\hline
\end{tabular}




\begin{tabular}{|c|c|c|c|c|c|c|c|c|c|c|}
\hline No. & $\begin{array}{l}\text { Date } \\
\text { I } 901\end{array}$ & Gr. M. T. & Mags. & $\begin{array}{c}\text { Height at } \\
\text { beginning } \\
\text { miles }\end{array}$ & $\begin{array}{c}\text { Height at } \\
\text { ending } \\
\text { miles }\end{array}$ & $\begin{array}{l}\text { Length } \\
\text { of Path } \\
\text { miles }\end{array}$ & $\begin{array}{l}\text { Velocity } \\
\text { in miles } \\
\text { per sec. }\end{array}$ & $\mathrm{Rac}$ & diant & Observers \\
\hline Io & Aug. 15 & $10^{\mathrm{h}} 6^{1} / \mathrm{m}^{\mathrm{m}}$ & $4-3$ & 77 & $5^{8}$ & $3^{8}$ & 60 & $5^{6^{\circ}}$ & $+5^{\circ}$ & J. H. B., W. F. D. \\
\hline I I & $\gg$ & I0 48 & $4-9$ & 81 & $6 x$ & 37 & $4 I$ & $5^{2}$ & +53 & J. H. B., A. King \\
\hline I 2 & Aug. 17 & 10 I 2 & $3-2$ & 72 & 66 & $27 \%$ & 45 & $5^{8}$ & +34 & A. S. H., W. F. D. \\
\hline I 3 &, & I 15 & $2 / 2-2$ & 58 & 53 & 22 & 32 & 95 & +46 & A. S. H., C. L. Brook \\
\hline I 4 & Aug. 18 & $1324 \%$ & $4-3$ & 62 & 50 & 28 & I 5 & 106 & +52 & A. S. H., W.F.D. \\
\hline I 5 & Aug. 19 & $1341 \%$ & $2-2$ & 64 & 50 & 18 & 28 & 337 & +3 & A. S. H., W. F. D. \\
\hline 16 & Aug. 20 & 1114 & $>4$ & $5^{6}$ & 33 & 44 & I 3 & 2 I 3 & +49 & C. J. Spencer, W.F.D. \\
\hline 17 & » & 12 & $2-2$ & 64 & $5^{8}$ & 17 & 23 & 347 & -15 & A. S. H., W. F. D. \\
\hline 18 & Aug. 2 I & 1019 & $2 \rightarrow>I$ & 63 & $5^{2}$ & 20 & 25 & 341 & +5 & A. King, W.F.D. \\
\hline I 9 & $\triangleright$ & I $7 \%$ & $3-1$ & 71 & 60 & 34 & 40 & $5 \mathrm{I}$ & +27 & C. L. Brook, W. F. D. \\
\hline
\end{tabular}

Nos. 2 and 16 are remarkable for their very slow motions, the observed velocities being not more than half parabolic speed.

Nos. 3, 4, 6, 8, 9, to and I I were Perseids.

Nos. 2 and 17 were directed from an apparently new shower in Aquarius at about $350^{\circ}-14^{\circ}$.

The abbreviations in the last column headed,$o b-$ serverse are

\begin{tabular}{|c|c|c|c|c|c|c|c|c|c|}
\hline \multicolumn{2}{|c|}{1901} & \multicolumn{2}{|c|}{ Gr. M. T. } & \multirow{2}{*}{$\begin{array}{l}\text { Mag. } \\
>I\end{array}$} & \multicolumn{2}{|c|}{ From } & \multicolumn{2}{|l|}{ To } & \multirow{2}{*}{$\begin{array}{l}\text { Path } \\
25^{\circ}\end{array}$} \\
\hline July & 15 & $13^{h}$ & $5^{\mathrm{m}}$ & & $\stackrel{\alpha}{270^{\circ}}$ & $\begin{array}{c}\delta \\
+53^{\circ}\end{array}$ & ${ }_{24}^{\alpha} 8^{1 / 2^{\circ}}$ & $\begin{array}{c}\delta \\
+32^{\circ}\end{array}$ & \\
\hline July & 18 & 13 & 43 & 2 & 102 & $\begin{array}{r}33 \\
+64\end{array}$ & 90 & $+59 \%$ & 7 \\
\hline July & 19 & I I & $4 I$ & $\mathbf{I}$ & 335 & +68 & 289 & $+67 \%$ & $17^{1 / 2}$ \\
\hline July & 21 & 13 & 43 & 1 & $349^{1 / 8}$ & +15 & 341 & $+1 / 2$ & 16 \\
\hline Aug. & 10 & 11 & 34 & $\mathbf{I}$ & 55 & $+751 / 2$ & 217 & +75 & 29 \\
\hline * & & 12 & 0 & I & 32 & $+581 / 2$ & 24 & $+5^{8}$ & 4 \\
\hline 》 & & 12 & 47 & 24 & $33 \times 1 / 2$ & $+61 / 2$ & $324 \%$ & -6 & 15 \\
\hline$>$ & & 12 & 52 & 4 & $35^{6}$ & +57 & $359^{1 / 2}$ & $+3^{8}$ & I 9 \\
\hline » & & 13 & 0 & 4 & 33 & -4 & $3^{2}$ & -12 & 8 \\
\hline , & & 13 & $171 / 2$ & $>24$ & 333 & +60 & 302 & $+451 / 8$ & $221 /$ \\
\hline$\triangleright$ & & 13 & 33 & 1 & 62 & $+221 / 2$ & 66 & $+11 \%$ & 12 \\
\hline Aug. & 11 & 9 & $5^{\circ}$ & $\mathbf{I}$ & 320 & +16 & 307 & -4 & 23 \\
\hline$>$ & & 10 & 34 & $\mathbf{I}$ & I I & +10 & 6 & -4 & I 5 \\
\hline , & & I I & 2 & $2 \times 9$ & $353^{1 / 2}$ & +7 & 343 & -14 & 23 \\
\hline$»$ & & I I & 34 & 4 & 43 & +79 & 232 & +73 & 28 \\
\hline$>$ & & I I & $5^{6}$ & $q$ & 120 & +74 & 160 & +65 & 16 \\
\hline 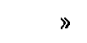 & & 12 & 5 & I & 29 & $+54 \%$ & $20 \%$ & $+501 \%$ & 6 \\
\hline Aug. & I 2 & 14 & 4 & 4 & 46 & -5 & 46 & -15 & ro \\
\hline Aug. & 16 & 13 & 11 & I & $\times 5$ & $+3^{8}$ & 7 & +28 & 12 \\
\hline Aug. & I 8 & I I & 29 & 2 & 3 & +53 & 285 & +45 & 48 \\
\hline Aug. & 19 & $1 \mathrm{I}$ & 49 & 2 & 347 & -12 & $35^{8}$ & $-81 / 2$ & $11 \%$ \\
\hline > & & 14 & 5 & 4 & 322 & $+4^{81 / 2}$ & $33^{81 / 2}$ & +41 & 14 \\
\hline Aug. & 20 & 9 & 37 & $>I$ & 359 & +23 & I & $+18 \%$ & 5 \\
\hline , & & 10 & 16 & $>I$ & 349 & +43 & $327^{1 / 2}$ & $+19 \frac{1}{2}$ & 29 \\
\hline$\triangleright$ & & I I & 8 & $>$ I & 307 & +39 & 302 & $+3^{2}$ & 8 \\
\hline * & & 11 & 14 & $>24$ & 2 I 4 & +70 & 2 I 8 & +84 & 14 \\
\hline Aug. & 21 & II & 9 & 2 & $89^{1 / 2}$ & $+71 \frac{1}{2}$ & 201 & $+68^{1} \%$ & 33 \\
\hline ' & & 13 & 27 & $>I$ & 302 & +49 & $312 \frac{1}{2}$ & +45 & 8 \\
\hline$>$ & & 13 & 37 & $>r$ & 42 & +2 & 68 & $+9 \%$ & $26^{1 / 2}$ \\
\hline Aug. & 22 & XI I & 29 & 2 & 224 & +65 & I 66 & $+67 \%$ & 221 \\
\hline
\end{tabular}

Bishopston, Bristol, r 90 I Sept. 13.
A.S.H. Prof. A. S. Herschel, Slough

J.H. B. J. H. Bridger, Farnborough

W. F.D. W. F. Denning, Bristol.

Two of the most important features ascertained from this years observations are that the Perseids certainly continue visible until Aug. $2 \mathrm{I}$ and that meteors differing greatly in their relative velocities diverge from the same radiants.

Bright meteors or meteors from radiants in unusual positions or little known were recorded at Bristol as follows:
Notes

rapid, streak slowish, streak v. rapid, streak v. rapid, streak v. rapid, streak rapid, streak v. rapid, streak slow, train v. rapid, streak v. rapid, streak v. v. slow, train rapid, streak rapid, streak rapid, streak rapid, streak rapid, streak slowish, streak rapid, streak rapid, streak rapid, streak v. v. slow v. slow, train rapid, train rapid, streak rapid

v. slow, train rapid, streak slow, train slow, train v. slow
Radiant

$29^{\circ}+36^{\circ}$ or Perseid

$133^{\circ}+69^{\circ}$

$29^{\circ}+36^{\circ}$ or Perseid

Perseid

$43^{\circ}+12^{\circ}$

Perseid

Perseid

$193^{\circ}+57^{\circ}$

Perseid

Perseid

$269^{\circ}+47^{\circ}$

Perseid

Perseid

Perseid

Perseid

Perseid

Perseid

Perseid

Perseid

$57^{\circ}+9^{\circ}$

$310-20$

$290+53$

$310+6 I$

Perseid

$329^{\circ}+61^{\circ}$

$213+49$

$57+9$

$290+53$

$350-14$

$283-12$

W. F. Denning. 